\title{
On global solutions to a nonlinear Alfvén wave equation
}

\author{
by XS. FENG and F. WeI (Beijing)
}

\begin{abstract}
We establish the global existence and uniqueness of smooth solutions to a nonlinear Alfvén wave equation arising in a finite-beta plasma. In addition, the spatial asymptotic behavior of the solution is discussed.
\end{abstract}

1. Introduction. The aim of this paper is to consider the Cauchy problem

$$
\begin{gathered}
\partial_{t} u+i u_{x x}=\alpha\left(u|u|^{2}\right)_{x}+\beta\left(u H|u|^{2}\right)_{x}, \quad t>0, x \in \mathbb{R}, \\
u(x, 0)=\phi(x),
\end{gathered}
$$

where $\alpha$ and $\beta$ are real constants, $\beta>0, i=\sqrt{-1}$ and

$$
(H f)(x)=\mathrm{P} . \mathrm{V} \cdot \int_{-\infty}^{\infty} \frac{f(y)}{\pi(y-x)} d y
$$

denotes the Hilbert transform.

In [11], considering the effects of finite-temperature, Mjølhus and Wyller derived equation (1.1) from a set of equations describing weakly nonlinear, weakly dispersive, weakly diffractive and unidirectional MHD waves propagating nearly parallel to the ambient magnetic field in a finite-beta collisionless plasma. The nonlinear nonlocal term in (1.1) represents the effect of a resonant particle.

When $\beta=0$, equation (1.1) reduces to the well-known derivative nonlinear Schrödinger (DNLS) equation. Kaup and Newell [7] obtained an exact solution for the DNLS equation by using an appropriate version of the inverse scattering method. Hayashi [5] discussed the Cauchy problem for the DNLS equation by means of nonlinear transforms. Tsutsumi and Fukuda $[14,15]$ studied the DNLS equation

$$
u_{t}+i u_{x x}=\left(u|u|^{k}\right)_{x},
$$

1991 Mathematics Subject Classification: 35Q20, 35D99.

Key words and phrases: nonlinear Alfvén wave, existence and uniqueness of global solution, spatial asymptotic behavior. 
where $k$ is a natural number. In particular, Kenig et al. [8] established a local well-posedness result for the initial value problem for a general nonlinear Schrödinger equation of the form

$$
u_{t}=i \Delta u+P\left(u, \nabla_{x} u, \bar{u}, \nabla_{x} \bar{u}\right), \quad t \in \mathbb{R}, x \in \mathbb{R}^{n},
$$

where $u=u(x, t)$ is a complex-valued function, $P: \mathbb{C}^{2 n+2} \rightarrow \mathbb{C}$ is a polynomial having no constant or linear terms and $\nabla_{x} u=\left(\partial_{x_{1}} u, \ldots, \partial_{x_{n}} u\right)$.

For a detailed introduction to the Cauchy problem for the nonlinear Schrödinger equations, one can refer to [8] and references therein. It should be pointed out that the method developed in [8] is not applicable to equation (1.1). One cannot expect to obtain similar estimates (such as (4.8) of [8]) as done in [8] because of the appearance of the nonlocal term in (1.1). Thus, in the present paper, we shall employ the well-known parabolic regularization method in order to establish the global existence of solutions to problem (1.1), (1.2).

This paper is divided into four parts including the introduction. Section 2 gives some preliminaries and announces the results of this paper. Sections 3 and 4 contain the proofs of the results.

2. Preliminaries and results. Here we give the notation that will be used throughout this paper and announce the results of this paper.

As usual, $L^{p}(\mathbb{R}), 1 \leq p \leq \infty$, and $H^{s}(\mathbb{R}), s \in \mathbb{R}$, are the usual Lebesgue and Sobolev spaces with norms $|\cdot|_{p}$ and $\|\cdot\|_{s}$, respectively. If $I$ is an interval and $X$ a Banach space with norm $\|\cdot\|_{X}$, then $L^{p}(I ; X) \equiv\{u: I \rightarrow X$ : $\left.\|u\|_{X} \in L^{p}(I)\right\} . W_{p}^{r}\left(0, T ; H^{k}(\mathbb{R})\right)$ denotes the space of functions $f(x, t)$ that have derivatives $\partial_{t}^{s} \partial_{x}^{h} f(t, x) \in L^{p}\left(0, T ; L^{2}(\mathbb{R})\right)$ with $0 \leq s \leq r, 0 \leq h \leq k$. We denote by $C, C(\cdot, \cdot, \cdot)$ generic constants, not necessarily the same at each occurrence, which depend in an increasing way on the indicated quantities.

Let $Z=\{0,1,2, \ldots\}, \omega(x)=\left(1+x^{2}\right)^{1 / 2}$ and $S(\mathbb{R})$ be the Schwartz space of all rapidly decreasing infinitely differentiable functions on $\mathbb{R}$. Then for any $s, r \in \mathbb{R}, H_{r}^{s}$ is the completion of $S(\mathbb{R})$ under the norm $\|u\|_{r, s}=\mid \omega^{r}(1-$ $\left.\partial_{x}^{2}\right)\left.^{s / 2} u\right|_{2}$. Let $J_{r}^{s}=H_{r}^{0} \cap H_{0}^{s}$ with the norm $\|u\|_{r, s}=\left(\|u\|_{r, 0}^{2}+\|u\|_{0, s}^{2}\right)^{1 / 2}$. For $H_{r}^{s}$ and $J_{r}^{s}$ we have $[3,13]$

LEMMA 2.1. (a) $H_{r^{\prime}}^{s^{\prime}} \subseteq H_{r}^{s}, J_{r^{\prime}}^{s^{\prime}} \subseteq J_{r}^{s}, s \leq s^{\prime}, r \leq r^{\prime}$.

(b) $\left[H_{r_{1}}^{s_{1}}, H_{r_{2}}^{s_{2}}\right]_{\theta}=H_{(1-\theta) r_{1}+\theta r_{2}}^{(1-\theta) s_{1}+\theta s_{2}}, 0<\theta<1, s_{j}, r_{j} \in \mathbb{R}, j=1,2$, where $[,]_{\theta}$ denotes the complex interpolation.

(c) $J_{r}^{s} \subseteq\left[H_{r}^{0}, H_{0}^{s}\right]_{\theta}=H_{(1-\theta) r}^{\theta s}, 0<\theta<1, s, r \in \mathbb{R}$.

(d) $\bigcap_{r, s \in Z} J_{r}^{s}=\bigcap_{r, s \in Z}=S(\mathbb{R})$.

(e) $\left(J_{r}^{s}\right)^{\prime}=H_{-r}^{0}+H_{0}^{-s}$.

(f) Let $r, s>0$. If $u \in J_{r}^{r s}$, then $u \in H_{r-1}^{s}$ and $\|u\|_{r-1, s} \leq C(r, s)\|u\| \|_{r, r s}$. 
(g) Let $r \in \mathbb{R}$ and $s \geq 0$. Then for $h \in Z$ we have

$$
\left\|\partial_{x}^{h} u\right\|_{r, s} \leq C(r, s)\|\| u \|_{r^{\prime}, s^{\prime}},
$$

where $r^{\prime}=(s+h) r / s$ and $s^{\prime}=s+h$.

(h) If $s>1 / 2$, then for all $u, v \in J_{r}^{s}$ we have

$$
\|u v\|_{r, s} \leq C(r, s)\|\| u \mid\|\|_{r, s}\|v\|_{r, s}
$$

R e m a r k. By the lemma above and Sobolev embedding theorem we see that if $u \in J_{r}^{r(s+1)}$ with $s, r \in Z$ and $r \neq 0$, then

$$
\sup _{x \in \mathbb{R}}\left|(\omega(x))^{r-1} \partial_{x}^{s} u(x)\right| \leq C(r, s)\|u\|_{r-1, s+1} \leq C(r, s)\|u\| \|_{r,(s+1) r},
$$

that is, $\left|\partial_{x}^{s} u(x)\right|=O\left(|x|^{-(r-1)}\right)$ as $|x| \rightarrow \infty$.

The following statement follows from a straightforward calculation and its proof is omitted here.

LEMMA 2.2. Let $\mu(x) \in C_{0}^{\infty}(\mathbb{R})$ be such that $0 \leq \mu \leq 1, \mu=1$ if $|x| \leq 1$ and $\mu=0$ if $|x| \geq 2$. Let $\mu_{\varepsilon}(x)=\mu(\varepsilon x)$ for $0<\varepsilon<1$. Then as $\varepsilon \rightarrow 0$,

$$
\begin{array}{ll}
\mu_{\varepsilon}(x) \rightarrow 1 & \text { uniformly on any bounded set of } \mathbb{R}, \\
\partial_{x}^{j} \mu_{\varepsilon}(x) \rightarrow 0 & \text { uniformly on } \mathbb{R} \text { for } j \neq 0 .
\end{array}
$$

Moreover, for any $j \in Z$ we have

$$
\left|\partial_{x}^{h}(x)\right| \leq C(j) \varepsilon^{h}(\omega(x))^{-(j-h)}, \quad 0 \leq h \leq j,
$$

where $C(j)>0$ is independent of $\varepsilon$.

LEMMA 2.3. Let $q$ and $r$ be any real numbers satisfying $1 \leq q, r \leq \infty$ and $j, m \in Z$ be such that $j \leq m$. Then for $u \in L^{q}$ with $\partial_{x}^{m} u \in L^{r}$ we have

$$
\left|\partial_{x}^{j} u\right|_{p} \leq C(j, m, q, r, a)\left|\partial_{x}^{m} u\right|_{r}^{a}|u|_{q}^{1-a},
$$

where $1 / p=j+a(1 / r-m)+(1-a) 1 / q$ for all $a$ in the interval $j / m \leq a$ $\leq 1$. In particular,

for $u \in H^{1}$.

$$
|u|_{\infty} \leq|u|_{2}^{1 / 2}\left|u_{x}\right|_{2}^{1 / 2}
$$

The first result in this lemma is well known. For the second conclusion we know that

$$
u^{2}=2 \int_{-\infty}^{x} u u_{x} d x, \quad u^{2}=-2 \int_{x}^{\infty} u u_{x} d x
$$

From the above two equations we have

$$
|u|^{2} \leq 2 \int_{-\infty}^{x}\left|u u_{x}\right| d x \text { and }|u|^{2} \leq 2 \int_{x}^{\infty}\left|u u_{x}\right| d x .
$$


Adding the above two inequalities yields

$$
|u|^{2} \leq\left(\int_{-\infty}^{x}+\int_{x}^{\infty}\right)\left|u u_{x}\right| d x=\int_{-\infty}^{\infty}\left|u u_{x}\right| d x
$$

which implies the result by Hölder's inequality.

LEMMA 2.4 (Gronwall's inequality). Suppose that $g(t)$ and $h(t)$ satisfy the inequality

$$
g(t) \leq M_{1}+M_{2} \int_{0}^{t} g(s) h(s) d s \quad \text { for any } 0 \leq t \leq T,
$$

where $M_{1}$ and $M_{2}$ are nonnegative constants. Moreover, suppose that $\int_{0}^{T} h(t) d t<\infty$. Then

$$
g(t) \leq M_{1} \exp \left(M_{2} \int_{0}^{T} h(t) d t\right), \quad t \in[0, T] .
$$

LEMma 2.5 (Properties of Hilbert transform). (a) If $f, g \in L^{2}(\mathbb{R})$, then

$$
\int f H g d x=-\int g H f d x, \quad \int H f H g d x=\int f g d x .
$$

(b) For real functions $g \in H^{2}$, we have

1) $(H g)_{x}=H\left(g_{x}\right)$ and $\int g_{x} H(g) d x \geq 0$,

2) $\left|g_{x}\right|_{2} \leq\left(\int g_{x} H(g) d x\right)^{1 / 4}\left(\int g_{x x} H\left(g_{x}\right) d x\right)^{1 / 4}$, and

$$
\left|g_{x}\right|_{2} \leq|g|_{2}^{1 / 3}\left(\int g_{x x} H\left(g_{x}\right) d x\right)^{2 / 3} .
$$

Part (a) and 1) of part (b) are well known. The conclusion 2) of (b) can be proved directly by using Fourier transform and the Plancherel formula [2].

Our results are as follows:

ThEOREM 2.6. Let $T$ be any given positive constant. For any initial data $\phi \in H^{s}, s \in Z$ and $s \geq 2$, if

$$
|\phi|_{2}^{2}<\frac{4}{3\left(\sqrt{(|\alpha|+\beta)^{2}+\frac{8}{3} \alpha^{2}}+|\alpha|+\beta\right)}
$$

then problem (1.1), (1.2) has a solution u such that

$$
u \in \bigcap_{k+2 h \leq s} W_{\infty}^{h}\left(0, T ; H^{k}(\mathbb{R})\right)
$$

where $k, h \in Z$. 
THEOREM 2.7. Let $T$ be any given positive constant. For any initial data $\phi \in J_{r}^{s}(\mathbb{R})$ satisfying the condition of Theorem 2.6 with $s, r \in Z$ and $s \geq \max (r, 2)$, problem (1.1), (1.2) has a solution $u$ such that

$$
u \in \bigcap_{s^{\prime}+2 h \leq s, r^{\prime} s+2 h r \leq r s} W_{\infty}^{h}\left(0, T ; J_{r^{\prime}}^{s^{\prime}}\right),
$$

where $s^{\prime}, h \in Z$ and $r^{\prime} \geq 0$ real.

COROllary 2.8. For any $T>0$ fixed, if $\phi \in S(\mathbb{R})$ satisfies the condition of Theorem 2.6, then the solution u to problem (1.1), (1.2) belongs to $C^{\infty}([0, T] ; S(\mathbb{R}))$.

Corollary 2.9. Let $u_{\alpha, \beta}$ be the solution obtained in Theorems 2.6 and 2.7. Then as $\beta \rightarrow 0$ (resp. $\alpha \rightarrow 0$ ), $u_{\alpha, \beta}$ converges in

$$
\bigcap_{k+2 h \leq s} W_{\infty}^{h}\left(0, T ; H^{k}\right) \quad\left(\text { resp. } \bigcap_{s^{\prime}+2 h \leq s, r^{\prime} s+2 h r \leq r s} W_{\infty}^{h}\left(0, T ; J_{r^{\prime}}^{s^{\prime}}\right)\right)
$$

to the unique solution to the equations

$$
u_{t}+i u_{x x}=\alpha\left(|u|^{2} u\right)_{x}
$$

and

respectively.

$$
u_{t}+i u_{x x}=\beta\left(u H|u|^{2}\right)_{x}
$$

3. Proof of Theorem 2.6. In the study of global existence for dispersive equations, global a priori estimates play an important role. It is a common technique to use the conservation laws to establish global a priori estimates. But in our case we do not know explicit forms of the conservation laws associated with equation (1.1). In this section we derive global a priori estimates of the solution to (1.1), (1.2) by constructing substitutes for the conservation laws corresponding to (1.1). In order to prove Theorem 2.6 we first consider the initial value problem for the following parabolic regularization:

$$
\begin{gathered}
u_{t}+i u_{x x}=\varepsilon u_{x x}+\alpha\left(u|u|^{2}\right)_{x}+\beta\left(u H|u|^{2}\right)_{x}, \quad t>0, \varepsilon>0, x \in \mathbb{R}, \\
u(x, 0)=\phi^{\varepsilon}(x), \quad \varepsilon>0,
\end{gathered}
$$

where $\phi^{\varepsilon}(x) \in H^{\infty}(\mathbb{R})=\bigcap_{k>0} H^{k}(\mathbb{R})$ with $\phi^{\varepsilon}(x) \rightarrow \phi(x)$ strongly in $H^{s}(\mathbb{R})$ as $\varepsilon \downarrow 0+$. Then the solution to problem (1.1), (1.2) is obtained by the standard limiting process $\varepsilon \downarrow 0+$.

For problem (3.1), (3.2) we have

Lemma 3.1. For any given $T>0$, there exists a unique solution $u^{\varepsilon}$ to problem (3.1), (3.2) such that $u^{\varepsilon} \in C^{\infty}\left([0, T] ; H^{\infty}(\mathbb{R})\right)$. 
P r o of. The proof is simple. By using the argument of [9] one can establish the existence in $L^{\infty}\left(0, T ; L^{2}\right) \cap L^{2}\left(0, T ; H^{1}\right)$. Then differentiating the equation with respect to $x$ one obtains the $L^{\infty}\left(0, T ; H^{1}\right) \cap L^{2}\left(0, T ; H^{2}\right)$ regularity. Continuing in this way and applying the standard results for linear parabolic problems $[1,10]$ one can finish the proof.

In order to guarantee the convergence of the limiting process we need uniform estimates of the solution to problem (3.1), (3.2). The uniform estimates are established in the subsequent several lemmas.

LEMMA 3.2. For the solution $u=u^{\varepsilon}$ obtained in Lemma 3.1 we have the following identities:

1) $\frac{d}{d t} \int|u|^{2} d x+\beta \int\left(|u|^{2}\right)_{x} H|u|^{2} d x+2 \varepsilon \int\left|u_{x}\right|^{2} d x=0$,

2) $\frac{d}{d t} \int\left[\left|u_{x}\right|^{2}+\frac{3}{2} \alpha \operatorname{Im}\left(\bar{u} u_{x}\right)|u|^{2}+\frac{3}{2} \beta \operatorname{Im}\left(\bar{u} u_{x}\right) H|u|^{2}+\frac{3}{2} \alpha^{2}|u|^{6}\right] d x$

$=\beta\left[6 \alpha \operatorname{Im} \int u_{x} \bar{u}|u|^{2} H\left(|u|^{2}\right)_{x} d x+\frac{3}{2} \alpha \operatorname{Im} \int \bar{u} u_{x}\left(|u|^{2}\right)_{x} H|u|^{2} d x\right.$

$+\frac{3}{2} \beta \operatorname{Im} \int \bar{u} u_{x} H|u|^{2} H\left(|u|^{2}\right)_{x} d x-\frac{3}{2} \alpha \operatorname{Im} \int\left(\bar{u} H|u|^{2}\right)_{x}\left(u|u|^{2}\right)_{x} d x$

$+\frac{3}{2} \alpha \operatorname{Im} \int \bar{u} u_{x} H\left(|u|^{2}\left(|u|^{2}\right)_{x}\right) d x+3 \beta \operatorname{Im} \int \bar{u} u_{x} H\left(|u|^{2} H\left(|u|^{2}\right)_{x}\right) d x$

$\left.+\frac{3}{2} \beta \operatorname{Im} \int \bar{u} u_{x} H\left(\left(|u|^{2}\right)_{x} H|u|^{2}\right) d x-\frac{5}{2} \alpha^{2} \int|u|^{6} H\left(|u|^{2}\right)_{x} d x\right]$

$+\varepsilon \int\left[3 \alpha^{2}|u|^{4} \operatorname{Re}\left(\bar{u} u_{x x}\right)+6 \alpha \operatorname{Im}\left(u_{x} \bar{u}_{x x}\right)|u|^{2}\right] d x$

$+\frac{3}{2} \beta \varepsilon \operatorname{Im} \int\left[\left(u_{x} \bar{u}_{x x}\right) H|u|^{2}-\left(\bar{u} H|u|^{2}\right)_{x} u_{x x}+2\left(\bar{u} u_{x}\right) H\left(\operatorname{Re}\left(\bar{u} u_{x x}\right)\right)\right] d x$.

Proof. The proof of 1) can be given by multiplying (3.1) by $\bar{u}^{\varepsilon}$, integrating the resulting expression in $x$ and performing several integrations by parts. The proof of 2) can be established directly by integration by parts. In fact, using equation (3.1) and integration by parts we have

$$
\frac{d}{d t} \int\left|u_{x}\right|^{2} d x+2 \varepsilon \int\left|u_{x x}\right|^{2} d x
$$

$=3 \beta \int\left|u_{x}\right|^{2} H\left(|u|^{2}\right)_{x} d x-\beta \int\left(|u|^{2}\right)_{x x} H\left(|u|^{2}\right)_{x} d x+3 \alpha \int\left(|u|^{2}\right)_{x}\left|u_{x}\right|^{2} d x$,

$$
\begin{aligned}
& \frac{d}{d t} \int \operatorname{Im}\left(\bar{u} u_{x}\right)|u|^{2} d x \\
& =-2 \int\left(|u|^{2}\right)_{x}\left|u_{x}\right|^{2} d x+4 \varepsilon \int \operatorname{Im}\left(u_{x} \bar{u}_{x x}\right)|u|^{2} d x \\
& \quad+4 \alpha \int \operatorname{Im}\left(\bar{u} u_{x}\right)|u|^{2}\left(|u|^{2}\right)_{x} d x+4 \beta \int \operatorname{Im}\left(\bar{u} u_{x}\right)|u|^{2} H\left(|u|^{2}\right)_{x} d x
\end{aligned}
$$

$$
\frac{d}{d t} \int \operatorname{Im}\left(\bar{u} u_{x}\right) H|u|^{2} d x
$$




$$
\begin{aligned}
& =-2 \int H\left(|u|^{2}\right)_{x}\left|u_{x}\right|^{2} d x+\frac{1}{2} \int\left(|u|^{2}\right)_{x x x} H\left(|u|^{2}\right)_{x x} d x \\
& +2 \int \operatorname{Im}\left(\bar{u} u_{x}\right) H\left(\operatorname{Im}\left(\bar{u} u_{x x}\right)\right) d x \\
& +\varepsilon \operatorname{Im} \int\left(u_{x} \bar{u}_{x x} H|u|^{2}-\left(\bar{u} H|u|^{2}\right)_{x} u_{x x}+2\left(\bar{u} u_{x}\right) H\left(\operatorname{Re}\left(\bar{u} u_{x x}\right)\right)\right) d x \\
& +\alpha \int \operatorname{Im}\left(\bar{u} u_{x}\right)\left(|u|^{2}\right)_{x} H|u|^{2} d x+\beta \int \operatorname{Im}\left(\bar{u} u_{x}\right) H|u|^{2} H\left(|u|^{2}\right)_{x} d x \\
& -\alpha \operatorname{Im} \int\left(\bar{u} H|u|^{2}\right)_{x}\left(u|u|^{2}\right)_{x} d x+3 \alpha \int \operatorname{Im}\left(\bar{u} u_{x}\right) H\left(|u|^{2}\left(|u|^{2}\right)_{x}\right) d x \\
& +\beta \int \operatorname{Im}\left(\bar{u} u_{x}\right)\left(2 H\left(|u|^{2} H\left(|u|^{2}\right)_{x}\right)+H\left(\left(|u|^{2}\right)_{x} H|u|^{2}\right)\right) d x, \\
& \frac{d}{d t} \int|u|^{6}=-4 \int|u|^{2}\left(|u|^{2}\right)_{x} \operatorname{Im}\left(\bar{u} u_{x}\right) d x-\frac{5}{3} \beta \int|u|^{6} H\left(|u|^{2}\right)_{x} d x \\
& \quad+2 \varepsilon \int|u|^{4} \operatorname{Re}\left(\bar{u} u_{x x}\right) d x .
\end{aligned}
$$

Considering (3.3)-(3.6) we can complete the proof.

From this lemma we get the following conclusion:

LEMma 3.3. Under the conditions of Lemma 3.1, if

$$
|\phi|_{2}^{2}<\frac{4}{3\left(\sqrt{(|\alpha|+\beta)^{2}+\frac{8}{3} \alpha^{2}}+|\alpha|+\beta\right)},
$$

then

$$
\|u(\cdot, t)\|_{1}^{2}+\varepsilon \int_{0}^{t}\|u(\cdot, \tau)\|_{2}^{2} d \tau \leq C
$$

and

$$
\frac{\beta}{4} \int_{0}^{t} \int\left(|u|^{2}\right)_{x x} H\left(|u|^{2}\right)_{x} d x d \tau+3 \beta \int_{0}^{t} \int \operatorname{Im}\left(\bar{u} u_{x}\right)_{x} H \operatorname{Im}\left(\bar{u} u_{x}\right) d x d \tau \leq C
$$

hold for all $t \geq 0$, where $C$ is a positive constant depending only on the size of $\|\phi\|_{1}$.

Proof. From 1) of Lemma 3.2 it follows that

$$
|u(\cdot, t)|_{2}^{2}+\beta \int_{0}^{t} \int\left(|u|^{2}\right)_{x} H|u|^{2} d x+2 \varepsilon \int_{0}^{t}\left|u_{x}(\cdot, \tau)\right|_{2}^{2} d \tau \leq C
$$

for all $t \geq 0$. Denoting the right hand side of 2 ) in Lemma 3.2 by $A(t)$ and using Lemmas 2.3 and 2.5 we have the following delicate estimate:

$$
\begin{aligned}
|A(t)| \leq & C \beta\left(|u|_{\infty}^{3}\left|u_{x}\right|_{2}\left|\left(|u|^{2}\right)_{x}\right|_{2}\right. \\
& \left.+\left.\left.\left|u_{x}\right|_{2}\left|\left(|u|^{2}\right)_{x}\right|_{2}|u|_{\infty}|H| u\right|^{2}\right|_{\infty}+|u|_{\infty}^{4} \|\left.\left. u\right|^{2}\right|_{2}\left|\left(|u|^{2}\right)_{x}\right|_{2}\right) \\
& +C \varepsilon\left(|u|_{\infty}^{4}|u|_{2}\left|u_{x x}\right|_{2}+\left|u_{x}\right|_{\infty}|u|_{4}^{2}\left|u_{x x}\right|_{2}+|u|_{\infty}^{2}\left|u_{x}\right|_{2}\left|u_{x x}\right|_{2}\right)
\end{aligned}
$$




$$
\begin{aligned}
\leq & C \beta\left(|u|_{\infty}^{3}\left|u_{x}\right|_{2}\left|\left(|u|^{2}\right)_{x}\right|_{2}\right. \\
& \left.+\left.\left.\left|u_{x}\right|_{2}\left|\left(|u|^{2}\right)_{x}\right|_{2}|u|_{\infty}|H| u\right|^{2}\right|_{\infty}+|u|_{\infty}^{4} \|\left.\left. u\right|^{2}\right|_{2}\left|\left(|u|^{2}\right)_{x}\right|_{2}\right) \\
& +C \varepsilon\left(|u|_{2}^{3}\left|u_{x}\right|_{2}^{2}\left|u_{x x}\right|_{2}+|u|_{2}^{3 / 2}\left|u_{x}\right|_{2}\left|u_{x x}\right|_{2}^{3 / 2}\right) .
\end{aligned}
$$

In order to estimate the first term on the right hand side of (3.8) we use the following inequalities (see Lemma 2.3):

$$
\|\left.\left. u\right|^{2}\right|_{\infty} \leq\left.\left. C\left|\left(|u|^{2}\right)_{x}\right|_{2}^{2 / 3}|| u\right|^{2}\right|_{1} ^{1 / 3}
$$

and

$$
\left.\left.\left\|\left.\left.u\right|^{2}\right|_{2} \leq C\left|\left(|u|^{2}\right)_{x}\right|_{2}^{1 / 3}\right\| u\right|^{2}\right|_{1} ^{2 / 3}
$$

From (3.9), (3.10) we have

$$
|u|_{\infty}^{3}=\|\left.\left. u\right|^{2}\right|_{\infty} ^{3 / 2} \leq C\left|\left(|u|^{2}\right)_{x}\right|_{2}|u|_{2}
$$

and

$$
\begin{aligned}
\left.\left.|u|_{\infty}|H| u\right|^{2}\right|_{\infty} & \leq|u|_{\infty}\left|\left(|u|^{2}\right)_{x}\right|_{2}^{1 / 2} \|\left.\left. u\right|^{2}\right|_{2} ^{1 / 2} \\
& =\left.\left.|| u\right|^{2}\right|_{\infty} ^{1 / 2} \|\left.\left. u\right|^{2}\right|_{2} ^{1 / 2}\left|\left(|u|^{2}\right)_{x}\right|_{2}^{1 / 2} \leq C|u|_{2}\left|\left(|u|^{2}\right)_{x}\right|_{2}, \\
|u|_{\infty}^{4} \|\left.\left. u\right|^{2}\right|_{2} & =|u|_{\infty}^{3}|u|_{\infty}|u|_{4}^{2} \leq C|u|_{2}^{3}\left|u_{x}\right|_{2}\left|\left(|u|^{2}\right)_{x}\right|_{2} .
\end{aligned}
$$

Inserting (3.11)-(3.13) into (3.8) we obtain

$$
\begin{aligned}
|A(t)| \leq & C \beta\left(|u|_{2}\left|u_{x}\right|_{2}+|u|_{2}^{3}\left|u_{x}\right|_{2}\right)\left|\left(|u|^{2}\right)_{x}\right|_{2}^{2} \\
& +C \varepsilon\left(|u|_{2}^{3}\left|u_{x}\right|_{2}^{2}\left|u_{x x}\right|_{2}+|u|_{2}^{3 / 2}\left|u_{x}\right|_{2}\left|u_{x x}\right|_{2}^{3 / 2}\right) \\
\leq & C \beta\left|u_{x}\right|_{2}\left|\left(|u|^{2}\right)_{x}\right|_{2}^{2}+C \varepsilon\left(\left|u_{x}\right|_{2}^{2}\left|u_{x x}\right|_{2}+\left|u_{x}\right|_{2}\left|u_{x x}\right|_{2}^{3 / 2}\right) \\
\leq & C \beta\left|u_{x}\right|_{2}\left(\int\left(|u|^{2}\right)_{x} H|u|^{2} d x\right)^{1 / 2}\left(\int\left(|u|^{2}\right)_{x x} H\left(|u|^{2}\right)_{x} d x\right)^{1 / 2} \\
& +\varepsilon\left|u_{x x}\right|_{2}^{2}+C \varepsilon\left|u_{x}\right|_{2}^{4} \\
\leq & \frac{1}{8} \beta \int\left(|u|^{2}\right)_{x x} H\left(|u|^{2}\right)_{x} d x+C \beta\left(\int\left(|u|^{2}\right)_{x} H|u|^{2} d x\right)\left|u_{x}\right|_{2}^{2} \\
& +\varepsilon\left|u_{x x}\right|_{2}^{2}+C \varepsilon\left|u_{x}\right|_{2}^{4},
\end{aligned}
$$

where we have used Lemma 2.5 and Young's inequality. Consequently, we get

(3.15) $\frac{d}{d t} \int\left[\left|u_{x}\right|^{2}+\frac{3}{2} \beta \operatorname{Im}\left(\bar{u} u_{x}\right) H|u|^{2}+\frac{3}{2} \alpha \operatorname{Im}\left(\bar{u} u_{x}\right)|u|^{2}+\frac{3}{2} \alpha^{2}|u|^{6}\right] d x$

$+\frac{1}{8} \beta \int\left(|u|^{2}\right)_{x x} H\left(|u|^{2}\right)_{x} d x+3 \beta \int\left(\operatorname{Im}\left(\bar{u} u_{x}\right)\right)_{x} H\left(\operatorname{Im}\left(\bar{u} u_{x}\right)\right)+\varepsilon \int\left|u_{x x}\right|^{2} d x$

$$
\leq C\left(\beta \int\left(|u|^{2}\right)_{x} H|u|^{2} d x+\varepsilon\left|u_{x}\right|_{2}^{2}\right)\left|u_{x}\right|_{2}^{2} .
$$

To bound (3.5), first note that

$$
\begin{aligned}
& \left.\left.\left|\frac{3}{2} \beta \int \operatorname{Im}\left(\bar{u} u_{x}\right) H\right| u\right|^{2} d x\left|\leq \frac{3}{2} \beta\right| \phi\right|_{2} ^{2}\left|u_{x}\right|_{2}^{2}, \\
& \left.\left|\frac{3}{2} \alpha \int \operatorname{Im}\left(\bar{u} u_{x}\right) H\right| u\right|^{2} d x\left|\leq \frac{3}{2}\right| \alpha \|\left.\phi\right|_{2} ^{2}\left|u_{x}\right|_{2}^{2},
\end{aligned}
$$




$$
\frac{3}{2} \alpha^{2} \int|u|^{6} d x \leq \frac{3}{2} \alpha^{2}\left|u_{x}\right|_{2}^{2}|u|_{2}^{4} \leq \frac{3}{2} \alpha^{2}|\phi|_{2}^{4}\left|u_{x}\right|_{2}^{2} .
$$

Keeping (3.16)-(3.18) in mind and integrating (3.15) over the time interval $[0, t]$ we obtain

$$
\begin{aligned}
& \left|u_{x}\right|_{2}^{2}+\frac{1}{8} \beta \int_{0}^{t} \int\left(|u|^{2}\right)_{x x} H\left(|u|^{2}\right)_{x} d x d t \\
& +3 \beta \int_{0}^{t} \int\left(\operatorname{Im}\left(\bar{u} u_{x}\right)\right)_{x} H\left(\operatorname{Im}\left(\bar{u} u_{x}\right)\right) d x d t+\varepsilon \int_{0}^{t} \int\left|u_{x x}\right|^{2} d x d t \\
& \leq C+C \int_{0}^{t}\left(\beta \int\left(|u|^{2}\right)_{x} H|u|^{2} d x+\varepsilon\left|u_{x}\right|_{2}^{2}\right)\left|u_{x}\right|_{2}^{2} d \tau
\end{aligned}
$$

if $|\phi|_{2}^{2}$ is less than the positive root of the algebraic equation

that is,

$$
\frac{3}{2} \alpha^{2} y^{2}+\frac{3}{2}(|\alpha|+\beta) y-1=0,
$$

$$
|\phi|_{2}^{2}<\frac{4}{3\left(\sqrt{(|\alpha|+\beta)^{2}+\frac{8}{3} \alpha^{2}}+|\alpha|+\beta\right)} .
$$

Now apply Gronwall's inequality to (3.19) and use (3.7) to finish the proof.

Lemma 3.4. Under the conditions of Lemma 3.3, we have

$$
\begin{aligned}
& \left|u_{x x}\right|_{2}^{2}+\varepsilon \int_{0}^{t}\left|u_{x x x}\right|_{2}^{2} d \tau \leq C, \\
& \beta \int_{0}^{t} \int\left(|u|^{2}\right)_{x x x} H\left(|u|^{2}\right)_{x x} d x d \tau \\
& \quad+\beta \int_{0}^{t} \int \operatorname{Im}\left(\bar{u} u_{x}\right)_{x x} H \operatorname{Im}\left(\bar{u} u_{x}\right)_{x} d x d \tau \leq C
\end{aligned}
$$

for all $t \in[0, T]$, where $C$ depends only on $T$ and $\|\phi\|_{H^{2}}$.

Proof. Using equation (3.1) and integration by parts we can obtain the following somewhat tedious identities:

$$
\begin{aligned}
& (3.21) \quad \frac{d}{d t} \int\left|u_{x x}\right|^{2} d x \\
& =5 \alpha \int\left(|u|^{2}\right)_{x}\left|u_{x x}\right|^{2} d x+5 \alpha \int\left(|u|^{2}\right)_{x x}\left(\left|u_{x}\right|^{2}\right)_{x} d x \\
& \quad+\beta \int\left(5\left|u_{x x}\right|^{2} H\left(|u|^{2}\right)_{x}+5 H\left(|u|^{2}\right)_{x x}\left(\left|u_{x}\right|^{2}\right)_{x}-\left(|u|^{2}\right)_{x x x} H\left(|u|^{2}\right)_{x x}\right) d x \\
& (3.22) \quad \frac{d}{d t} \int \operatorname{Im}\left(u_{x} \bar{u}_{x x}\right) H|u|^{2} d x \\
& \quad=2 \int H\left(|u|^{2}\right)_{x}\left|u_{x x}\right|^{2} d x+\frac{1}{2} \int H\left(|u|^{2}\right)_{x x}\left(\left|u_{x}\right|^{2}\right)_{x} d x
\end{aligned}
$$


$+2 \int \operatorname{Im}\left(u_{x} \bar{u}_{x x}\right) H\left(\operatorname{Im}\left(\bar{u} u_{x}\right)_{x}\right) d x$

$+\varepsilon \operatorname{Im} \int\left[\bar{u}_{x x} u_{x x x} H|u|^{2}-\left(u_{x} H|u|^{2}\right)_{x} \bar{u}_{x x x}\right.$

$\left.+2 u_{x} \bar{u}_{x x} H\left(\operatorname{Re}\left(\bar{u} u_{x x}\right)\right)\right] d x$

$+\alpha \operatorname{Im} \int \bar{u}_{x x}\left(u|u|^{2}\right)_{x x} H|u|^{2} d x$

$+\beta \operatorname{Im} \int \bar{u}_{x x}\left(u H|u|^{2}\right)_{x x} H|u|^{2} d x$

$-\alpha \operatorname{Im} \int\left(u|u|^{2}\right)_{x}\left(\bar{u} H|u|^{2}\right)_{x x} d x-\beta \operatorname{Im} \int\left(u_{x} H|u|^{2}\right)_{x}\left(\bar{u} H|u|^{2}\right)_{x x} d x$

$+3 \alpha \operatorname{Im} \int u_{x} \bar{u}_{x x} H\left(\left(|u|^{2}\right)_{x}|u|^{2}\right) d x+2 \beta \operatorname{Im} \int u_{x} \bar{u}_{x x} H\left(|u|^{2} H\left(|u|^{2}\right)_{x}\right) d x$

$+\beta \operatorname{Im} \int u_{x} \bar{u}_{x x} H\left(\left(|u|^{2}\right)_{x} H|u|^{2}\right) d x$,

(3.23) $\frac{d}{d t} \int \operatorname{Im}\left(u \bar{u}_{x x x}\right) H|u|^{2} d x$

$=-2 \int H\left(|u|^{2}\right)_{x}\left|u_{x x}\right|^{2} d x-\frac{5}{2} \int H\left(|u|^{2}\right)_{x x}\left(\left|u_{x}\right|^{2}\right)_{x} d x$

$+\frac{1}{2} \int\left(|u|^{2}\right)_{x x x} H\left(|u|^{2}\right)_{x x} d x-2 \int\left(\operatorname{Im}\left(u \bar{u}_{x}\right)\right)_{x x} H\left(\operatorname{Im}\left(u \bar{u}_{x}\right)\right)_{x} d x$

$+2 \int \operatorname{Im}\left(u_{x} \bar{u}_{x x}\right) H\left(\operatorname{Im}\left(u \bar{u}_{x}\right)\right)_{x} d x$

$+\varepsilon \operatorname{Im} \int\left[u_{x x} \bar{u}_{x x x} H|u|^{2}+\left(u H|u|^{2}\right)_{x x} \bar{u}_{x x x}+2 u \bar{u}_{x x x} H\left(\operatorname{Re}\left(\bar{u} u_{x x}\right)\right)\right] d x$

$-4 \alpha \operatorname{Im} \int u \bar{u}_{x x} H\left(|u|^{2}\left(|u|^{2}\right)_{x}\right)_{x} d x-4 \alpha \operatorname{Im} \int u_{x} \bar{u}_{x x}\left(|u|^{2}\right)_{x} H|u|^{2} d x$

$-\alpha \operatorname{Im} \int\left(|u|^{2} H|u|^{2}\right)_{x} u_{x} \bar{u}_{x x} d x-3 \beta \operatorname{Im} \int H\left(|u|^{2}\right) H\left(|u|^{2}\right)_{x} u_{x} \bar{u}_{x x} d x$

$-\beta \operatorname{Im} \int\left(H\left(|u|^{2}\right) H\left(|u|^{2}\right)_{x}\right)_{x} u \bar{u}_{x x} d x$

$-2 \beta \operatorname{Im} \int u_{x} \bar{u}_{x x} H\left(|u|^{2} H\left(|u|^{2}\right)_{x}\right) d x$

$-2 \beta \operatorname{Im} \int u \bar{u}_{x x}\left(H\left(|u|^{2}\right) H\left(|u|^{2}\right)_{x}\right)_{x} d x$

$-\beta \operatorname{Im} \int u_{x} \bar{u}_{x x} H\left(\left(|u|^{2}\right)_{x} H|u|^{2}\right) d x$

$-\beta \operatorname{Im} \int u \bar{u}_{x x} H\left(\left(|u|^{2}\right)_{x} H|u|^{2}\right)_{x} d x$,

(3.24) $\frac{d}{d t} \int \operatorname{Im}\left(u_{x} \bar{u}_{x x}\right)|u|^{2} d x$

$$
=\int\left(|u|^{2}\right)_{x}\left|u_{x x}\right|^{2} d x+\int\left(|u|^{2}\right)_{x x}\left(\left|u_{x}\right|^{2}\right)_{x} d x
$$




$$
\begin{aligned}
& +\varepsilon \operatorname{Im} \int\left[|u|^{2} u_{x x} \bar{u}_{x x x}+2 u_{x} \bar{u}_{x x} \operatorname{Re}\left(\bar{u} u_{x x}\right)-\left(|u|^{2} u_{x}\right)_{x} \bar{u}_{x x x}\right] d x \\
& +\alpha \operatorname{Im} \int|u|^{2} \bar{u}_{x x}\left(u|u|^{2}\right)_{x x} d x+\beta \operatorname{Im} \int|u|^{2} \bar{u}_{x x}\left(u H|u|^{2}\right)_{x x} d x \\
& -\alpha \operatorname{Im} \int\left(|u|^{2} u_{x}\right)_{x}\left(\bar{u}|u|^{2}\right)_{x x} d x-\beta \operatorname{Im} \int\left(|u|^{2} u_{x}\right)_{x}\left(\bar{u} H|u|^{2}\right)_{x x} d x \\
& +3 \alpha \operatorname{Im} \int u_{x} \bar{u}_{x x}|u|^{2}\left(|u|^{2}\right)_{x} d x+2 \beta \operatorname{Im} \int u_{x} \bar{u}_{x x}|u|^{2} H\left(|u|^{2}\right)_{x} d x \\
& +\beta \operatorname{Im} \int u_{x} \bar{u}_{x x}\left(|u|^{2}\right)_{x} H|u|^{2} d x
\end{aligned}
$$

Considering (3.21)-(3.24) we get

$$
\begin{aligned}
\frac{d}{d t} \int\left[\left|u_{x x}\right|^{2}\right. & +\frac{15}{8} \beta \operatorname{Im}\left(u \bar{u}_{x x x}\right) H|u|^{2} \\
& \left.-\frac{5}{8} \beta \operatorname{Im}\left(u_{x} \bar{u}_{x x}\right) H|u|^{2}-5 \alpha \operatorname{Im}\left(u_{x} \bar{u}_{x x}\right)|u|^{2}\right] d x \\
& +2 \varepsilon \int\left|u_{x x x}\right|^{2} d x+\frac{1}{16} \beta \int\left(|u|^{2}\right)_{x x x} H\left(|u|^{2}\right)_{x x} d x \\
& +\frac{15}{4} \beta \int\left(\operatorname{Im}\left(u \bar{u}_{x}\right)\right)_{x x} H\left(\operatorname{Im}\left(u \bar{u}_{x}\right)\right)_{x} d x \\
\leq & C \beta\left|u_{x}\right|_{\infty}\left|u_{x x}\right|_{2}\left|\operatorname{Im}\left(u \bar{u}_{x}\right)_{x}\right|_{2}+\left(1+\left|u_{x x}\right|_{2}^{2}\right)+\varepsilon\left|u_{x x x}\right|_{2}^{2},
\end{aligned}
$$

where we have used Lemmas 2.3 and 2.5 and Young's inequality.

Using Lemma 2.5 we have

$$
\begin{aligned}
\left|\left(\operatorname{Im}\left(u \bar{u}_{x}\right)\right)_{x}\right|_{2}^{2} \leq & \left(\int\left(\operatorname{Im}\left(u \bar{u}_{x}\right)\right)_{x} H\left(\operatorname{Im}\left(u \bar{u}_{x}\right)\right) d x\right)^{1 / 2} \\
& \times\left(\int\left(\operatorname{Im}\left(u \bar{u}_{x}\right)\right)_{x x} H\left(\operatorname{Im}\left(u \bar{u}_{x}\right)\right)_{x} d x\right)^{1 / 2}
\end{aligned}
$$

Combining (3.25) and (3.26) yields that the right hand side of (3.25) is less than

$$
\begin{aligned}
C \beta\left|u_{x x}\right|_{2}^{3 / 2}\left(\int\left(\operatorname{Im}\left(u \bar{u}_{x}\right)\right)_{x} H\left(\operatorname{Im}\left(u \bar{u}_{x}\right)\right) d x\right)^{1 / 4} \\
\times\left(\int\left(\operatorname{Im}\left(u \bar{u}_{x}\right)\right)_{x x} H\left(\operatorname{Im}\left(u \bar{u}_{x}\right)\right)_{x} d x\right)^{1 / 4} \\
+C\left(1+\left|u_{x x}\right|_{2}^{2}\right)+\varepsilon\left|u_{x x x}\right|_{2}^{2} \\
\leq C+C\left(1+\beta \int\left(\operatorname{Im}\left(u \bar{u}_{x}\right)\right)_{x} H\left(\operatorname{Im}\left(u \bar{u}_{x}\right)\right) d x\right)\left|u_{x x}\right|_{2}^{2}+\varepsilon\left|u_{x x x}\right|_{2}^{2} \\
\quad+\beta \int\left(\operatorname{Im}\left(u \bar{u}_{x}\right)\right)_{x x} H\left(\operatorname{Im}\left(u \bar{u}_{x}\right)\right)_{x} d x .
\end{aligned}
$$

By integration by parts and Lemma 2.3 we have 
(3.28) $\left.\left|\frac{15}{8} \beta \operatorname{Im} \int \bar{u} u_{x x x} H\right| u\right|^{2} d x$

$$
\begin{aligned}
&-\frac{5}{8} \beta \operatorname{Im} \int u_{x} \bar{u}_{x x} H|u|^{2} d x-5 \alpha \operatorname{Im} \int u_{x} \bar{u}_{x x}|u|^{2} d x \mid \\
& \leq C\left|u_{x x}\right|_{2} \leq \frac{1}{2}\left|u_{x x}\right|_{2}^{2}+C .
\end{aligned}
$$

From (3.25), (3.27) and (3.28) we get

$$
\begin{aligned}
\left|u_{x x}\right|_{2}^{2} & +\frac{1}{16} \beta \int_{0}^{t} \int\left(|u|^{2}\right)_{x x x} H\left(|u|^{2}\right)_{x x} d x d t+\varepsilon \int_{0}^{t} \int\left|u_{x x x}\right|^{2} d x d t \\
& +\frac{11}{4} \beta \int_{0}^{t} \int\left(\operatorname{Im}\left(u \bar{u}_{x}\right)\right)_{x x} H\left(\operatorname{Im}\left(u \bar{u}_{x}\right)\right)_{x} d x d t \\
\leq & C+C \int_{0}^{t}\left(1+\beta \int\left(\operatorname{Im}\left(u \bar{u}_{x}\right)\right)_{x} H\left(\operatorname{Im}\left(u \bar{u}_{x}\right)\right) d x\right)\left|u_{x x}\right|_{2}^{2} d \tau
\end{aligned}
$$

The application of Gronwall's inequality to (3.29) completes the proof.

Lemma 3.5. Under the conditions of Lemma 3.3, for $k \leq s$ we have

$$
\left|u_{k x}\right|_{2}^{2}+\varepsilon \int_{0}^{t} \int\left|u_{(k+1) x}\right|^{2} d x d t+\beta \int_{0}^{t}\left(|u|^{2}\right)_{(k+1) x} H\left(|u|^{2}\right)_{k x} d x d t \leq C
$$

for all $t \in[0, T]$, where $C$ depends only on $T$ and $\|\phi\|_{k}$, and $u_{k x}$ denotes the $k$ th derivative of $u$ in $x$.

Pro of. A simple calculation gives

$$
\begin{aligned}
(3.30) & \frac{d}{d t} \int\left|u_{k x}\right|^{2} d x+2 \varepsilon \int\left|u_{(k+1) x}\right|^{2} d x+\beta \int\left(|u|^{2}\right)_{(k+1) x} H\left(|u|^{2}\right)_{k x} d x \\
= & -\beta \int\left|u_{k x}\right|^{2} H\left(|u|^{2}\right)_{x} d x-\alpha \int\left|u_{k x}\right|^{2}\left(|u|^{2}\right)_{x} d x \\
& +2 \beta \operatorname{Re} \int \bar{u}_{k x}\left[\left(u H|u|^{2}\right)_{(k+1) x}-u_{(k+1) x} H|u|^{2}-u H\left(|u|^{2}\right)_{(k+1) x}\right] d x \\
& +\beta \int\left(\left(|u|^{2}\right)_{k x}-2 \operatorname{Re}\left(\bar{u}_{k x} u\right)\right)_{x} H\left(|u|^{2}\right)_{k x} d x \\
& +2 \alpha \operatorname{Re} \int \bar{u}_{k x}\left[\left(u|u|^{2}\right)_{(k+1) x}-u_{(k+1) x}|u|^{2}-u\left(|u|^{2}\right)_{(k+1) x}\right] d x \\
& +\alpha \int\left[\left(|u|^{2}\right)_{k x}-2 \operatorname{Re}\left(\bar{u}_{k x} u\right)\right]_{x}\left(|u|^{2}\right)_{k x} d x \\
\leq & C\left(\left|H\left(|u|^{2}\right)_{x}\right|_{\infty}+\left|\left(|u|^{2}\right)_{x}\right|_{\infty}\right)\left|u_{k x}\right|_{2}^{2} \\
& +\left.C\left|u_{k x}\right|_{2}\left|\left(u H|u|^{2}\right)_{(k+1) x}-u_{(k+1) x} H\right| u\right|^{2}-\left.u H\left(|u|^{2}\right)_{(k+1) x}\right|_{2}
\end{aligned}
$$




$$
\begin{aligned}
& +\left.C\left|u_{k x}\right|_{2}\left|\left(u|u|^{2}\right)_{(k+1) x}-u_{(k+1) x}\right| u\right|^{2}-\left.u\left(|u|^{2}\right)_{(k+1) x}\right|_{2} \\
& +C\left|\left(|u|^{2}\right)_{k x}\right|_{2}\left|\left[\left(|u|^{2}\right)_{k x}-2 \operatorname{Re}\left(\bar{u}_{k x} u\right)\right]_{x}\right|_{2} \\
\leq & C\left(\left|H\left(|u|^{2}\right)_{x}\right|_{\infty}+\left|\left(|u|^{2}\right)_{x}\right|_{\infty}\right)\left|u_{k x}\right|_{2}^{2}+C\left|\left(|u|^{2}\right)_{k x}\right|_{2}\left|u_{x}\right|_{\infty}\left|u_{k x}\right|_{2} \\
& +C\left|u_{k x}\right|_{2}\left(|u|_{\infty}\left|\left(|u|^{2}\right)_{k x}\right|_{2}+\left|H\left(|u|^{2}\right)_{x}\right|_{\infty}\left|u_{k x}\right|_{2}\right) \leq C\left|u_{k x}\right|_{2}^{2}
\end{aligned}
$$

Now the application of Gronwall's inequality implies the conclusion of the lemma.

From equation (3.1) and Lemmas 3.2-3.5 we know that

$$
\left\|u_{t}^{\varepsilon}\right\|_{L^{2}\left(0, T ; H^{s-2}\right)} \leq C, \quad \sup _{0 \leq t \leq T}\left\|u^{\varepsilon}\right\|_{s} \leq C,
$$

where $C$ is independent of $\varepsilon$. By weak compactness, we can find a subsequence of $\left\{u^{\varepsilon}\right\}_{\varepsilon>0}$ (also denoted by $\left.\left\{u^{\varepsilon}\right\}\right)$ and $u \in L^{\varepsilon}\left(0, T ; H^{s}\right) \cap L^{2}(0, T$; $H^{s-2}$ ) such that

Evidently,

$$
\begin{array}{ll}
u^{\varepsilon} \rightarrow u & \text { weak-star in } L^{\infty}\left(0, T ; H^{s}\right), \\
u_{t}^{\varepsilon} \rightarrow u_{t} & \text { weakly in } L^{2}\left(0, T ; H^{s-2}\right) .
\end{array}
$$

$$
\alpha\left(u^{\varepsilon}\left|u^{\varepsilon}\right|^{2}\right)_{x}+\beta\left(u^{\varepsilon} H\left|u^{\varepsilon}\right|^{2}\right)_{x} \rightarrow \alpha\left(u|u|^{2}\right)_{x}+\beta\left(u H|u|^{2}\right)_{x} .
$$

So, the limit $u$ of $\left\{u^{\varepsilon}\right\}$ satisfies equation (1.1). By a classical result of Strauss [12] we know that $u$ is weakly continuous from $[0, T]$ into $H^{s}$ after a modification on a set with zero measure. Thus $u^{\varepsilon}(x, 0)=\phi^{\varepsilon} \rightarrow \phi(x)=u(x, 0)$. Continuing in this way and using equation (1.1) we conclude that

$$
u \in \bigcap_{k+2 r \leq s} W_{\infty}^{r}\left(0, T ; H^{k}\right) .
$$

In order to complete the proof of Theorem 2.6 it remains to show the uniqueness. To this end, let $u$ and $v$ be two solutions to problem (1.1), (1.2) with initial data $\phi$ and $\psi$, respectively. Then $w=u-v$ is a solution to the problem

$$
\begin{gathered}
w_{t}+i w_{x x}=\alpha\left(u|u|^{2}-v|v|^{2}\right)_{x}+\beta\left(u H|u|^{2}-v H|v|^{2}\right)_{x} \\
w(x, 0)=\phi-\psi
\end{gathered}
$$

Using equation (3.31) and integration by parts we get

$$
\begin{aligned}
\frac{d}{d t}|w|_{2}^{2}= & 2 \operatorname{Re} \int \bar{w} w_{t} d x \\
= & -2 \alpha \operatorname{Re} \int \bar{w}_{x}\left[w \frac{|u|^{2}+|v|^{2}}{2}+\frac{u+v}{2} \operatorname{Re}(\bar{w}(u+v))\right] d x \\
& -2 \beta \operatorname{Re} \int \bar{w}_{x}\left[w H\left(\frac{|u|^{2}+|v|^{2}}{2}\right)+\frac{u+v}{2} H \operatorname{Re}(\bar{w}(u+v))\right] d x
\end{aligned}
$$




$$
\begin{aligned}
= & \alpha \int|w|^{2}\left(\frac{|u|^{2}+|v|^{2}}{2}\right)_{x} d x+\alpha \int \operatorname{Re}\left(\bar{w}(u+v)_{x}\right) \operatorname{Re}(\bar{w}(u+v)) d x \\
& +\beta \int|w|^{2} H\left(\frac{|u|^{2}+|v|^{2}}{2}\right)_{x} d x \\
& -\beta \int \operatorname{Re}(\bar{w}(u+v))_{x} H \operatorname{Re}(\bar{w}(u+v)) d x \\
& +\beta \int \operatorname{Re}\left(\bar{w}(u+v)_{x}\right) H \operatorname{Re}(\bar{w}(u+v)) d x \\
\leq & C|w|_{2}^{2}-\beta \int \operatorname{Re}(\bar{w}(u+v))_{x} H \operatorname{Re}(\bar{w}(u+v)) d x \leq C|w|_{2}^{2} .
\end{aligned}
$$

By Gronwall's inequality it follows that

$$
|w(\cdot, t)|_{2}^{2} \leq|\phi-\psi|_{2}^{2} \exp (C T), \quad t \in[0, T],
$$

which implies the uniqueness.

4. Proof of Theorem 2.7. It is of interest to know that equation (1.1) has solutions decreasing faster than $H^{s}(\mathbb{R})$ convergence as $x$ tends to infinity, in particular, solutions in the Schwartz space $S(\mathbb{R})$ for each $t$, provided that its initial data is in $S(\mathbb{R})$. This can be realized by considering the initial value problem (1.1), (1.2) in the weighted Sobolev space $J_{r}^{s}(\mathbb{R})$. For the $J_{r}^{s}(\mathbb{R})$ convergence of solutions to problem $(1.1)$, (1.2) we have Theorem 2.7 and Corollary 2.8. In the proof of our result we employ the same method as in Tsutsumi [13]. In fact, the proof can be reproduced from [13]. In order to make the paper self-contained we present the proof as follows.

Since $S(\mathbb{R})$ is dense in $J_{r}^{s}(\mathbb{R})$, there exists a sequence $\left\{\phi^{k}\right\} \subset S(\mathbb{R})$ such that

$$
\left\{\phi^{k}\right\} \rightarrow \phi \quad \text { strongly in } J_{r}^{s}(\mathbb{R}) \text { as } k \rightarrow \infty .
$$

We first consider the parabolic regularization of equation (1.1): for $k \in Z \backslash 0$,

$$
u_{t}+i u_{x x}=\frac{1}{k} u_{x x}+\alpha\left(|u|^{2} u\right)_{x}+\beta\left(u H|u|^{2}\right)_{x}
$$

with

$$
u(x, 0)=\phi^{k}(x) .
$$

For problem (4.2), (4.3) we have

LEMma 4.1. For every fixed $k \in Z \backslash 0$, problem (4.2), (4.3) has a unique global solution $u^{k} \in C^{\infty}([0, T] ; S(\mathbb{R})), T>0$.

Proof. By the results of Section 3 we know that problem (4.2), (4.3) has a unique global solution $u^{k} \in C^{\infty}\left([0, T] ; H^{\infty}\right)$ and

$$
\sup _{0 \leq t \leq T}\left\|u^{k}(t)\right\|_{s}^{2}+\frac{1}{k} \int_{0}^{T}\left\|u^{k}(t)\right\|_{s+1}^{2} d t \leq C,
$$


where $C$ is independent of $k$ but depends on the size of $\phi$ and $T$. In order to prove the assertion of the lemma, it suffices to show that

$$
u^{k} \in L^{\infty}\left(0, T ; J_{r}^{0}(\mathbb{R})\right)
$$

for every $r \in Z$. The proof of (4.5) together with

$$
\partial_{x} u^{k} \in L^{2}\left(0, T ; J_{r}^{0}(\mathbb{R})\right)
$$

is done by induction on $r$. When $r=0$, this is obvious. Assume that the result is known for all values less than or equal to $r-1(r \geq 1)$. We prove it for $r$. Let $\mu(x)$ and $\mu_{\varepsilon}(x)$ be as given in Lemma 1.2. For simplicity we sometimes suppress $k$ in $u^{k}$. Differentiating $\left\|u^{k}(t)\right\|_{r, 0}^{2}$ with respect to $t$, using equation (4.2) and integrating by parts we have

$$
\begin{aligned}
(4.7) \frac{d}{d t}\left\|\mu_{\varepsilon} u(t)\right\|_{r, 0}^{2}= & -\frac{2}{k}\left\|\mu_{\varepsilon} u_{x}\right\|_{r, 0}^{2}+\frac{1}{k} \int\left(\mu_{\varepsilon}^{2} \omega^{2 r}\right)_{x}|u|^{2} \\
& +2 \operatorname{Re} \int\left(\mu_{\varepsilon} \omega^{2 r}\right)_{x} i \bar{u} u_{x} d x \\
& +3 \alpha \int \mu_{\varepsilon}^{2} \omega^{2 r}|u|^{2}\left(|u|^{2}\right)_{x} d x \\
& +2 \beta \int \mu_{\varepsilon}^{2} \omega^{2 r}|u|^{2} H\left(|u|^{2}\right)_{x} d x \\
& +\beta \int \mu_{\varepsilon}^{2} \omega^{2 r}\left(|u|^{2}\right)_{x} H|u|^{2} d x \\
\leq & -\frac{1}{k}\left\|\mu_{\varepsilon} u_{x}\right\|_{r, 0}^{2}+C(k)\left(\|u\|_{r, 0}^{2}+\|u\|_{r-1,0}^{2}+\left\|u_{x}\right\|_{r-1,0}^{2}\right),
\end{aligned}
$$

where we have used $\left|\partial_{x}^{d} \omega^{2 r}\right| \leq C(d)(\omega(x))^{2 r-d}$ and $\left|\partial_{x}^{j} \mu_{\varepsilon}(x)\right| \leq C(j)(\omega(x))^{-j}$. And $C$ is a positive constant independent of $\varepsilon$. We can rewrite (4.7) as

(4.9) $\frac{d}{d t}\left\|\mu_{\varepsilon} u\right\|_{r, 0}^{2}+\frac{1}{k}\left\|\mu_{\varepsilon} u_{x}(t)\right\|_{r, 0}^{2} \leq C(k)\left(\left\|\mu_{\varepsilon} u\right\|_{r, 0}^{2}+\|u\|_{r-1,0}^{2}+\left\|u_{x}\right\|_{r-1,0}^{2}\right)$.

Now Gronwall's inequality gives

$$
\begin{gathered}
\sup _{0 \leq t \leq T}\left\|\mu_{\varepsilon} u^{k}(t)\right\|_{r, 0}^{2} \leq C, \\
\int_{0}^{T}\left\|\mu_{\varepsilon} u_{x}^{k}(t)\right\|_{r, 0}^{2} d t \leq C,
\end{gathered}
$$

where $C$ is independent of $\varepsilon$. Therefore, $\left\{\mu_{\varepsilon} u^{k}\right\}$ remains in a bounded subset of $L^{\infty}\left(0, T ; J_{r}^{0}(\mathbb{R})\right)$. So, taking the limit as $\varepsilon \rightarrow 0$, we see that $\mu_{\varepsilon} u^{k} \rightarrow u^{k}$ weak-star in $L^{\infty}\left(0, T ; J_{r}^{0}(\mathbb{R})\right)$ and the assertions $(4.5)$, (4.6) hold for $r$ since $L^{\infty}\left(0, T ; J_{r}^{0}\right)=\left(L^{1}\left(0, T ; H_{-r}^{0}+L^{2}\right)\right)^{\prime}$. This ends the proof of the lemma.

Proof of Theorem 2.7. With Lemma 4.1 we consider the convergence of $u^{k}$ as $k \rightarrow \infty$. From Lemma 4.1 we know that for any $T>0$,

$$
\sup _{0 \leq t \leq T}\left\|u^{k}(t)\right\|_{0, s} \leq C
$$


holds for all integers $s$. From this it follows that $\left\{u^{k}\right\}$ forms a bounded subset of $L^{\infty}\left(0, T ; J_{0}^{s}(\mathbb{R})\right)$. We next show that for $r>0,\left\{u^{k}\right\}$ remains bounded in $L^{\infty}\left(0, T ; J_{r}^{0}(\mathbb{R})\right)$. In fact, we have

$$
\begin{aligned}
\frac{d}{d t}\left\|u^{k}(t)\right\|_{r, 0}^{2}= & 2 \operatorname{Re} \int \omega^{2 r} \bar{u} u_{t} d x \\
= & -\frac{2}{k}\left\|u_{x}\right\|_{r, 0}^{2}-\frac{2}{k} \operatorname{Re} \int 2 r \omega^{2(r-1)} x \bar{u} u_{x} d x \\
& +4 r \operatorname{Re} \int\left(4 r \omega^{2(r-1)} x i \bar{u} u_{x}\right. \\
& \left.+2 \alpha \omega^{2 r} \bar{u}\left(u|u|^{2}\right)_{x}+2 \beta \omega^{2 r} \bar{u}\left(u H|u|^{2}\right)_{x}\right) d x \\
\leq & -\frac{1}{k}\left\|u_{x}\right\|_{r, 0}^{2}+C\|u\|_{r, 0}^{2}+C\left\|u_{x}\right\|_{r-1,0}^{2} \\
\leq & -\frac{1}{k}\left\|u_{x}\right\|_{r, 0}^{2}+C\|u\|_{r, 0}^{2}+C\|u\|_{r, r}^{2} \\
\leq & -\frac{1}{k}\left\|u_{x}\right\|_{r, 0}^{2}+C\left(\|u\|_{r, 0}^{2}+1\right),
\end{aligned}
$$

where we have used the uniform boundedness of $\|u\|_{0, s}$ and $s \geq \max (r, 2)$. Thus

$$
\frac{d}{d t}\|u\|_{r, 0}^{2}+\frac{1}{k}\left\|u_{x}\right\|_{r, 0}^{2} \leq C\left(\|u\|_{r, 0}^{2}+1\right),
$$

where $C$ is independent of the natural number $k$. Integrating (4.13) with respect to $t$ and using Gronwall's inequality give

$$
\sup _{0 \leq t \leq T}\left\|u^{k}(t)\right\|_{r, 0} \leq C
$$

with the constant $C$ independent of $k$. From (4.12) and (4.14) it follows that $\left\{u^{k}\right\}$ forms a bounded sequence in $L^{\infty}\left(0, T ; J_{r}^{s}(\mathbb{R})\right)$. Hence, there exists a subsequence of $\left\{u^{k}\right\}$ (also denoted by $\left\{u^{k}\right\}$ ) and $u \in L^{\infty}\left(0, T ; J_{r}^{s}(\mathbb{R})\right)$ such that

$$
u^{k} \rightarrow u \quad \text { weak-star in } L^{\infty}\left(0, T ; J_{r}^{s}(\mathbb{R})\right) .
$$

Then it can be easily seen by the standard argument that $u$ is the desired solution of (1.1), (1.2) (see [9]). From Lemma 1.1(g) it is shown that

$$
u_{2 x} \in L^{\infty}\left(0, T ; J_{r^{\prime}}^{s^{\prime}}(\mathbb{R})\right),
$$

where $s^{\prime}=s-2$ and $r^{\prime}=r(s-2) / s$. Here, in view of equation (1.1) we can conclude that

$$
u_{t} \in L^{\infty}\left(0, T ; J_{r^{\prime}}^{s^{\prime}}(\mathbb{R})\right)
$$

with $s^{\prime}$ and $r^{\prime}$ as above. Continuing in this way and using equation (1.1) we obtain the conclusion of Theorem 2.7. 
Corollary 2.8 is just a consequence of Theorem 2.7 and Lemma 1.1(d) combined.

Corollary 2.9 can be seen from the process of proof of Theorems 2.6 and 2.7 .

R e m a r k. From Corollary 2.8 one knows that equation (1.1) has rapidly decreasing solutions if its initial data is in $S(\mathbb{R})$. This shows that the nonlocal nonlinear term in (1.1) does not affect the decay rate of its solutions. Thus the effect of the nonlocal nonlinear term in equation (1.1) is quite different from that of similar nonlocal terms in the Benjamin-Ono equation and the higher order Benjamin-Ono equation, in which the decay rate of the solutions is limited because of the presence of the nonlocal terms $H u_{x x}$ and $H\left(u^{2}\right)_{x x}([6,4])$.

\section{References}

[1] C. A. Bardos, Regularity theorem for parabolic equations, J. Funct. Anal. 7 (1971), 311-322.

[2] XS. Feng, The existence of global weak solutions for the equation of ion acoustic waves with Landau damping, Math. Appl. 7 (1994), 230-234 (in Chinese).

[3] - , The global Cauchy problem for a nonlinear Schrödinger equation, to appear.

[4] XS. Feng and Y. Han, On the Cauchy problem for the third order Benjamin-Ono equation, J. London Math. Soc., to appear.

[5] N. Hayashi, On the derivative Schrödinger equation, Phys. D 55 (1992), 14-36.

[6] R. J. Iorio, Jr., On the Cauchy problem for the Benjamin-Ono equation, Comm. Partial Differential Equations 11 (1986), 1031-1081.

[7] D. J. Kaup and A. C. Newell, An exact solution for a derivative nonlinear Schrödinger equation, J. Math. Phys. 19 (1978), 798-801.

[8] C. Kenig, G. Ponce and L. Vega, Small solutions to nonlinear Schrödinger equations, Ann. Inst. H. Poincaré Anal. Non Linéaire 10 (1993), 255-288.

[9] J. L. Lions, Quelques méthodes de résolutions des problèmes aux limites non linéaires, Gauthier-Villars, Paris, 1969.

[10] J. L. Lions et E. Magenes, Problèmes aux limites non homogènes et applications, Tome I, Dunod, Paris, 1968.

[11] E. Mjølhus and J. Wyller, Nonlinear Alfvén waves in a finite-beta plasma, J. Plasma Physics 40 (1988), 299-318.

[12] W. A. Strauss, On continuity of functions with values in various Banach spaces, Pacific J. Math. 19 (1966), 543-551.

[13] M. Tsutsumi, Weighted Sobolev spaces, and rapidly descreasing solutions of some nonlinear dispersive wave equations, J. Differential Equations 42 (1981), 260-281.

[14] M. Tsutsumi and I. Fukuda, On solutions of the derivative nonlinear Schrödinger equation, existence and uniqueness theorem, Funkcial. Ekvac. 23 (1980), 259-277. 
[15] M. Tsutsumi and I. Fukuda, On solutions of the derivative nonlinear Schrödinger equation, ibid. 24 (1981), 85-94.

NUMERICAL LABORATORY FOR HELIOSPHERIC PHYSICS

CHINESE ACADEMY OF SCIENCES

P.O. BOX 8701

BEIJING 100080, CHINA

Reçu par la Rédaction le 20.11.1994 